ESAIM: PROCEEDINGS, December 2012, Vol. 38, p. 241-256

F. Coquel, M. Gutnic, P. Helluy, F. Lagoutière, C. Rohde, N. Seguin, Editors

\title{
A SPLITTING METHOD FOR THE ISENTROPIC BAER-NUNZIATO TWO-PHASE FLOW MODEL
}

\author{
Frédéric Coquel ${ }^{1}$, Jean-Marc HÉrard ${ }^{2}$ and Khaled Saleh ${ }^{2,3}$
}

\begin{abstract}
In the present work, we propose a fractional step method for computing approximate solutions of the isentropic Baer-Nunziato two-phase flow model. The scheme relies on an operator splitting method corresponding to a separate treatment of fast propagation phenomena due to the acoustic waves on the one hand and slow propagation phenomena due to the fluid motion on the other. The scheme is proved to preserve positive values of the statistical fractions and densities. We also provide two test-cases that assess the convergence of the method.
\end{abstract}

Résumé. Nous proposons ici une méthode à pas fractionnaires pour le calcul de solutions approchées pour la version isentropique du modèle diphasique de Baer-Nunziato. Le schéma s'appuie sur un splitting de l'opérateur temporel correspondant à la prise en compte différenciée des phénomènes de propagation rapide dus aux ondes acoustiques et des phénomènes de propagation lente dus aux ondes matérielles. On prouve que le schéma permet de préserver des valeurs positives pour les taux statistiques de présence des phases ainsi que pour les densités. Deux cas tests numériques permettent d'illustrer la convergence de la méthode.

\section{INTRODUCTION}

The two-fluid approach is useful for a detailed investigation of some patterns occurring in gas-solid twophase flows, or alternatively in water-vapour flows such as those encountered in pressurised water reactors. In the latter framework, a classical situation corresponds to the prediction of the boiling crisis, where the flow is initially dominated by the liquid phase while the vapour phase is dilute. Actually, the two-fluid model proposed in $[2,6,8,9,11]$ is one suitable candidate that enables the computation of two-phase flows in which few bubbles are statistically present in a liquid phase. For other approaches relying on different assumptions, see $[4,14]$. Several schemes have already been proposed in the literature in order to build consistent and stable approximations of the Baer-Nunziato model, among which we may cite those relying on interface Riemann solvers (see for instance $[12,15-17]$ ) and other schemes relying on relaxation techniques (see for instance [1]).

However, one difficulty -among others- that immediately arises when computing approximations of the Baer-Nunziato model is due to the fact that the convective effects in this non-conservative model require accurate enough schemes; otherwise, the numerical approximations provided by standard solvers seem to be useless, and one reason for that failure is that the mix of "fast" waves corresponding with acoustic waves and "slow" waves associated with material velocities requires the development of schemes which should be accurate for quantities governed by either fast or slow waves. We suggest here a possible way to tackle this difficult problem, which is grounded on the use of a fractional step method. Before going further on, we recall that this idea has already been used earlier within the framework of Euler equations (see for instance [3]),

1 CNRS, UMR 7641, CMAP École Polytechnique, Palaiseau F-91128, France.

2 EDF R\&D, Département MFEE, Chatou F-78401, France.

${ }^{3}$ Université Pierre et Marie Curie Paris 6, UMR 7598, LJLL, Paris F-75005, France. e-mail: saleh@ann.jussieu.fr

(C) EDP Sciences, SMAI 2012 
but also for the Baer-Nunziato model (see [5]). Roughly speaking, a two-step algorithm is introduced in order to account for acoustic waves in the two-phase medium within the first step, while the second step handles material waves. In order to simplify the presentation, we will restrict in this paper to the barotropic version of the BN model, but the extension to the standard BN model is straightforward. Moreover, the numerical treatment of source terms will be disregarded, and we refer to relevant references for that topic [10].

Actually, the paper is organized as follows. In Section 1, we present the set of partial differential equations of the Baer-Nunziato two-phase flow model in the isentropic framework, and we recall its main mathematical properties. In Section 2, we propose an operator splitting method for this model, and we describe the numerical treatment of each step. Finally, Section 3 is devoted to the numerical experiments, where two test cases have been implemented with a mesh refinement procedure that proves the convergence of the method.

\section{The Baer-Nunziato two-Phase Flow MODEL AND its Mathematical PROPERTIES}

In the present work, we consider a model formulated in Eulerian coordinates where balance equations account for the evolution of mass and momentum of each phase. For compressible isentropic one-dimensional flows there are five unknowns that describe the evolution of the two-phase flow: the velocities of each phase $u_{k}$ (where $k \in\{1,2\}$ ), the densities of each phase $\rho_{k}$ and the phase fractions $\alpha_{k}$ (knowing that $\alpha_{1}+\alpha_{2}=1$ ). The isentropic version of the model -firstly introduced by Baer \& Nunziato- reads

$$
\begin{aligned}
& \partial_{t} \alpha_{1}+v_{I} \partial_{x} \alpha_{1}=\Theta_{p}\left(p_{1}-p_{2}\right), \\
& \partial_{t}\left(\alpha_{1} \rho_{1}\right)+\partial_{x}\left(\alpha_{1} \rho_{1} u_{1}\right)=0 \\
& \partial_{t}\left(\alpha_{1} \rho_{1} u_{1}\right)+\partial_{x}\left(\alpha_{1} \rho_{1} u_{1}^{2}+\alpha_{1} p_{1}\right)-p_{I} \partial_{x} \alpha_{1}=\Theta_{u}\left(u_{2}-u_{1}\right), \\
& \partial_{t}\left(\alpha_{2} \rho_{2}\right)+\partial_{x}\left(\alpha_{2} \rho_{2} u_{2}\right)=0 \\
& \partial_{t}\left(\alpha_{2} \rho_{2} u_{2}\right)+\partial_{x}\left(\alpha_{2} \rho_{2} u_{2}^{2}+\alpha_{2} p_{2}\right)-p_{I} \partial_{x} \alpha_{2}=\Theta_{u}\left(u_{1}-u_{2}\right),
\end{aligned}
$$

where $v_{I}$ and $p_{I}$ are the interfacial velocity and pressure for which one must provide closure laws as well as for the relaxation coefficients $\Theta_{u}$ and $\Theta_{p}$. One classical choice in the existing literature (see [10]) is

$$
\begin{aligned}
\Theta_{p} & =\frac{\alpha_{1} \alpha_{2}}{\tau_{p} \Pi_{0}}, \\
\Theta_{p} & =\frac{1}{\tau_{u}} \frac{\left(\alpha_{1} \rho_{1}\right)\left(\alpha_{2} \rho_{2}\right)}{\alpha_{1} \rho_{1}+\alpha_{2} \rho_{2}},
\end{aligned}
$$

where $\Pi_{0}$ has the dimension of a pressure, and $\tau_{p}$ and $\tau_{u}$ are two characteristic times of the pressure and velocity relaxation processes. For liquid-vapor applications, where the vapor phase is assumed to be dilute (we also refer to [2] where one of the phases is dilute), a meaningful choice for the pair of interfacial velocity and pressure is

$$
\left(v_{I}, p_{I}\right)=\left(u_{2}, p_{1}\right) .
$$

In this case, the index 1 refers to the liquid phase while the index 2 refers to the vapor phase. We also assume a barotropic pressure law for each phase $\rho_{k} \mapsto p_{k}\left(\rho_{k}\right), k \in\{1,2\}$ that can be deduced from the complete set of equations of the Baer-Nunziato model when assuming formally a constant entropy $s_{k}$ for each phase. We only consider a smooth dependence of $p_{k}\left(\rho_{k}\right)$ such that $p_{k}\left(\rho_{k}\right)>0, p_{k}^{\prime}\left(\rho_{k}\right)>0, p_{k}^{\prime \prime}\left(\rho_{k}\right)+\frac{2}{\rho_{k}} p_{k}^{\prime}\left(\rho_{k}\right)>0$, $\lim _{\rho_{k} \rightarrow 0} p_{k}\left(\rho_{k}\right)=0$, and $\lim _{\rho_{k} \rightarrow+\infty} p_{k}\left(\rho_{k}\right)=+\infty$. We denote

$$
\mathbb{U}=\left(\alpha_{1}, \alpha_{1} \rho_{1}, \alpha_{1} \rho_{1} u_{1}, \alpha_{2} \rho_{2}, \alpha_{2} \rho_{2} u_{2}\right)
$$

the unknown vector which is expected to belong to the natural physical space

$$
\Omega=\left\{\mathbb{U}=\left(\alpha_{1}, \alpha_{1} \rho_{1}, \alpha_{1} \rho_{1} u_{1}, \alpha_{2} \rho_{2}, \alpha_{2} \rho_{2} u_{2}\right) \underset{242}{\mathbb{R}^{5}}, 0<\alpha_{k}<1, \rho_{k}>0, k \in\{1,2\}, \alpha_{1}+\alpha_{2}=1\right\} .
$$


System (1) takes the following condensed form

$$
\partial_{t} \mathbb{U}+\partial_{x} \mathbf{F}(\mathbb{U})+\mathbf{C}(\mathbb{U}) \partial_{x} \mathbb{U}=\mathbf{S}(\mathbb{U}), \quad x \in \mathbb{R}, t>0,
$$

where

$$
\mathbf{F}(\mathbb{U})=\left[\begin{array}{c}
0 \\
\alpha_{1} \rho_{1} u_{1} \\
\alpha_{1} \rho_{1} u_{1}^{2}+\alpha_{1} p_{1}\left(\rho_{1}\right) \\
\alpha_{2} \rho_{2} u_{2} \\
\alpha_{2} \rho_{2} u_{2}^{2}+\alpha_{2} p_{2}\left(\rho_{2}\right)
\end{array}\right], \quad \mathbf{C}(\mathbb{U}) \partial_{x} \mathbb{U}=\left[\begin{array}{c}
u_{2} \partial_{x} \alpha_{1} \\
0 \\
-p_{1} \partial_{x} \alpha_{1} \\
0 \\
-p_{1} \partial_{x} \alpha_{2}
\end{array}\right], \quad \mathbf{S}(\mathbb{U})=\left[\begin{array}{c}
\Theta_{p}\left(p_{1}\left(\rho_{1}\right)-p_{2}\left(\rho_{2}\right)\right) \\
0 \\
\Theta_{u}\left(u_{2}-u_{1}\right) \\
0 \\
\Theta_{u}\left(u_{1}-u_{2}\right)
\end{array}\right]
$$

The following proposition holds:

Proposition 1.1. For every state vector $\mathbb{U}$ in $\Omega$, the convective part of system (7) admits the following real eigenvalues:

$$
\sigma_{1}(\mathbb{U})=u_{2}, \quad \sigma_{2}(\mathbb{U})=u_{1}-c_{1}, \quad \sigma_{3}(\mathbb{U})=u_{1}+c_{1}, \quad \sigma_{4}(\mathbb{U})=u_{2}-c_{2}, \quad \sigma_{5}(\mathbb{U})=u_{2}+c_{2},
$$

where

$$
c_{1}=\sqrt{p_{1}^{\prime}\left(\rho_{1}\right)}, \quad c_{2}=\sqrt{p_{2}^{\prime}\left(\rho_{2}\right)}
$$

are the speeds of sound is each phase. The system is hyperbolic (i.e. the corresponding family of right eigenvectors spans $\mathbb{R}^{5}$ ) if and only if $u_{2} \neq u_{1}+c_{1}$ and $u_{2} \neq u_{1}-c_{1}$. In addition, the fields associated with the eigenvalues $\left\{\sigma_{i}\right\}_{i=2 . .5}$ are genuinely non linear while the field associated with $\sigma_{1}$ is linearly degenerate.

Proof. The proof follows from classical calculations that are left to the reader.

\section{A Splitting method for the Baer-Nunziato model}

Let us introduce the following operator splitting method for the Baer-Nunziato equations. It consists in separating the wave propagation phenomena according to their respective propagation speed.

The first step corresponds to the propagation of acoustic waves due to pressure and phase fraction disequilibrium:

$$
\begin{aligned}
& \partial_{t} \alpha_{1}=0, \\
\left(\mathscr{S}_{1}\right) \quad & \partial_{t} \alpha_{k} \rho_{k}=0, \\
& \partial_{t} \alpha_{k} \rho_{k} u_{k}+\partial_{x} \alpha_{k} p_{k}-p_{1} \partial_{x} \alpha_{k}=0 .
\end{aligned} \quad k \in\{1,2\}
$$

The second step considers the propagation of material waves due to the fluid motion:

$$
\begin{array}{ll} 
& \partial_{t} \alpha_{1}+u_{2} \partial_{x} \alpha_{1}=0, \\
& \partial_{t} \alpha_{k} \rho_{k}+\partial_{x} \alpha_{k} \rho_{k} u_{k}=0, \\
& \partial_{t} \alpha_{k} \rho_{k} u_{k}+\partial_{x} \alpha_{k} \rho_{k} u_{k}^{2}=0 .
\end{array} \quad k \in\{1,2\}
$$

Finally, the third step takes into account the relaxation terms

$$
\begin{aligned}
& \partial_{t} \alpha_{1}=\Theta_{p}\left(p_{1}-p_{2}\right), \\
\left(\mathscr{S}_{3}\right) \quad & \partial_{t} \alpha_{k} \rho_{k}=0, \\
& \partial_{t} \alpha_{k} \rho_{k} u_{k}=\Theta_{u}\left(u_{3-k}-u_{k}\right) .
\end{aligned} \quad k \in\{1,2\}
$$

Observe that the splitting steps $\left(\mathscr{S}_{1}\right)$ and $\left(\mathscr{S}_{2}\right)$ are an extension to the two-phase flow model of the work performed in [3] in the framework of Euler's equations. In the present work, we focus on physical configurations for which the characteristic times $\tau_{p}$ and $\tau_{u}$ of the relaxation terms are much larger than the simulation time $T$. As a consequence, we do not treat this last step $\left(\mathscr{S}_{3}\right)$ in the present paper, and we refer to [10] for the numerical treatment of these terms. From this point, we assume that $\mathbf{S}(\mathbb{U})=0$. 


\subsection{Numerical approximation}

In this section, we use the operator splitting method in order to derive a fractional-step numerical scheme, the aim being to approximate the weak solutions of a Cauchy problem associated with the homogeneous part of system (7):

$$
\left\{\begin{array}{l}
\partial_{t} \mathbb{U}+\partial_{x} \mathbf{F}(\mathbb{U})+\mathbf{C}(\mathbb{U}) \partial_{x} \mathbb{U}=0, \quad x \in \mathbb{R}, t>0, \\
\mathbb{U}(x, 0)=\mathbb{U}_{0}(x)
\end{array}\right.
$$

Let $\Delta t$ be the time step and $\Delta x$ the space step, which we assume here to be constant for simplicity in the notations. The space is partitioned into cells

$$
\mathbb{R}=\bigcup_{j \in \mathbb{Z}} C_{j} \quad \text { with } \quad C_{j}=\left[x_{j-\frac{1}{2}}, x_{j+\frac{1}{2}}[, \quad \forall j \in \mathbb{Z},\right.
$$

where $x_{j+\frac{1}{2}}=\left(j+\frac{1}{2}\right) \Delta x$ are the cell interfaces. At the discrete times $t^{n}=n \Delta t$, the solution of (11) is approximated on each cell $C_{j}$ by a constant value denoted by

$$
\mathbb{U}_{j}^{n}=\left(\left(\alpha_{1}\right)_{j}^{n},\left(\alpha_{1} \rho_{1}\right)_{j}^{n},\left(\alpha_{1} \rho_{1} u_{1}\right)_{j}^{n},\left(\alpha_{2} \rho_{2}\right)_{j}^{n},\left(\alpha_{2} \rho_{2} u_{2}\right)_{j}^{n}\right)^{T} .
$$

Before giving the precise description of the fractional step method, we state the following result which summarizes the main properties of the scheme:

Theorem 2.1. Under some natural CFL restriction (see (35) and (49)), the fractional step numerical scheme presented in this paper has the following properties:

(i) It preserves the maximum principle on the phase fractions $\alpha_{k}$, in the sense that

$$
\forall n \in \mathbb{N}, \quad\left(0<\alpha_{k, j}^{n}<1, \forall j \in \mathbb{Z}\right) \quad \Longrightarrow \quad\left(0<\alpha_{k, j}^{n+1}<1, \forall j \in \mathbb{Z}\right)
$$

(ii) It preserves positive values of the densities in the sense that

$$
\forall n \in \mathbb{N}, \quad\left(\rho_{k, j}^{n}>0, \forall j \in \mathbb{Z}\right) \Longrightarrow\left(\rho_{k, j}^{n+1}>0, \forall j \in \mathbb{Z}\right),
$$

(iii) The discretization of the partial masses $\alpha_{k} \rho_{k}$ is conservative,

(iv) The discretization of the total momentum $\alpha_{1} \rho_{1} u_{1}+\alpha_{2} \rho_{2} u_{2}$ is conservative.

Proof. The result follows from Propositions 2.4 and 2.5 stated in sections 2.2 and 2.3 below.

In the following two sections, we describe the fractional-step procedure associated with the time operator -splitting method in order to calculate the values of the approximate solution at time $t^{n+1},\left(\mathbb{U}_{j}^{n+1}\right)_{j \in \mathbb{Z}}$ from those at time $t^{n}$. Section 2.2 displays the numerical treatment of the Lagrangian step $\left(\mathscr{S}_{1}\right)$ while section 2.3 deals with the material transport step $\left(\mathscr{S}_{2}\right)$.

\subsection{Treatment of the first step}

In this section, we consider the numerical treatment of the following set of PDE's.

$$
\begin{aligned}
& \partial_{t} \alpha_{1}=0, \\
&\left(\mathscr{S}_{1}\right) \quad \partial_{t} \alpha_{k} \rho_{k}=0, \\
& \partial_{t} \alpha_{k} \rho_{k} u_{k}+\partial_{x} \alpha_{k} p_{k}-p_{1} \partial_{x} \alpha_{k}=0 .
\end{aligned}
$$

One can check that all the eigenvalues of this non conservative system are zero, which implies that no numerical method relying on the spectral radius of the Jacobian matrix (such as Rusanov's scheme) can be 
applied in the present case. Therefore we choose to treat this first step with a relaxation scheme. For this purpose, we introduce the following relaxation system which relaxes towards $\left(\mathscr{S}_{1}\right)$ in the limit $\varepsilon \rightarrow 0$ :

$$
\begin{aligned}
& \partial_{t} \alpha_{1}=0, \\
& \partial_{t} \alpha_{k} \rho_{k}=0, \\
& \partial_{t} \alpha_{k} \rho_{k} u_{k}+\partial_{x} \alpha_{k} \pi_{k}-\pi_{1} \partial_{x} \alpha_{k}=0, \\
& \partial_{t} \alpha_{k} \rho_{k} \pi_{k}+a_{k}^{2} \partial_{x} \alpha_{k} u_{k}-a_{k}^{2} u_{2} \partial_{x} \alpha_{k}=\frac{1}{\varepsilon} \alpha_{k} \rho_{k}\left(p_{k}-\pi_{k}\right) .
\end{aligned}
$$

$\pi_{k}$ is an additional unknown which relaxes towards the actual pressure $p_{k}$ as $\varepsilon \rightarrow 0$ and whose evolution is governed by the additional PDE (15). The numbers $a_{k}>0$ are two numerical parameters that need to be taken large enough so as to ensure the stability of the relaxation approximation in the regime of small $\varepsilon$. Typically, $a_{k}$ must follow the so-called Whitham condition:

$$
a_{k}^{2}>\max _{\tau_{k}}\left(-\frac{\partial p_{k}}{\partial \tau_{k}}\left(\tau_{k}\right)\right), \quad k=1,2
$$

where the max is taken over all the specific volumes $\tau_{k}$ in the solution of (12)-(15). We refer to [1] and [7] for a related framework.

Let us now focus on the convective part of this relaxation system which reads:

$$
\begin{aligned}
& \partial_{t} \alpha_{1}=0, \\
& \partial_{t} \alpha_{k} \rho_{k}=0, \\
\left(\mathscr{S}_{1} \mathscr{R}\right) \quad & \partial_{t} \alpha_{k} \rho_{k} u_{k}+\partial_{x} \alpha_{k} \pi_{k}-\pi_{1} \partial_{x} \alpha_{k}=0, \\
& \partial_{t} \alpha_{k} \rho_{k} \pi_{k}+a_{k}^{2} \partial_{x} \alpha_{k} u_{k}-a_{k}^{2} u_{2} \partial_{x} \alpha_{k}=0 .
\end{aligned}
$$

We have the following property on the characteristic fields of the relaxation system.

Proposition 2.2. For all state vector $\mathbb{W}=\left(\alpha_{1}, \alpha_{1} \rho_{1}, \alpha_{1} \rho_{1} u_{1}, \alpha_{1} \rho_{1} \pi_{1}, \alpha_{2} \rho_{2}, \alpha_{2} \rho_{2} u_{2}, \alpha_{2} \rho_{2} \pi_{2}\right)$ such that $\rho_{1}>0$ and $\rho_{2}>0$, system $\left(\mathscr{S}_{1} \mathscr{R}\right)$ has the following eigenvalues:

$$
-a_{k} \tau_{k}, \quad 0, \quad a_{k} \tau_{k}, \quad k \in\{1,2\},
$$

where $\tau_{k}=\rho_{k}^{-1}$ is the specific volume of phase $k$. Moreover, all the characteristic fields are linearly degenerate and system $\left(\mathscr{S}_{1} \mathscr{R}\right)$ is hyperbolic in the sense that the corresponding family of eigenvectors spans the whole space $\mathbb{R}^{7}$.

Proof. The proof is left to the reader.

Thus, the solution of a Riemann problem for $\left(\mathscr{S}_{1} \mathscr{R}\right)$ consists in six constant states separated by five contact discontinuities. The calculation of such a solution is easy since the jump relations across each contact discontinuity are given by the Riemann invariants of the corresponding wave. In the following array, we display the Riemann invariants for each wave:

\begin{tabular}{|c|c|}
\hline Wave's velocity & Riemann invariants \\
\hline$-a_{1} \tau_{1}$ & $\alpha_{1}, \rho_{1}, \rho_{2}, u_{2}, \pi_{2}, \pi_{1}+a_{1} u_{1}$ \\
\hline$-a_{2} \tau_{2}$ & $\alpha_{1}, \rho_{1}, \rho_{2}, u_{1}, \pi_{1}, \pi_{2}+a_{2} u_{2}$ \\
\hline 0 & $\alpha_{1} u_{1}+\alpha_{2} u_{2}, \alpha_{1} \pi_{1}+\alpha_{2} \pi_{2}, u_{2}, \pi_{1}$ \\
\hline$a_{2} \tau_{2}$ & $\alpha_{1}, \rho_{1}, \rho_{2}, u_{1}, \pi_{1}, \pi_{2}-a_{2} u_{2}$ \\
\hline$a_{1} \tau_{1}$ & $\alpha_{1}, \rho_{1}, \rho_{2}, u_{2}, \pi_{2}, \pi_{1}-a_{1} u_{1}$ \\
\hline
\end{tabular}

And we have the following proposition: 
Proposition 2.3. Let be given two initial states $\mathbb{W}_{L}$ and $\mathbb{W}_{R}$ such that $\rho_{1}>0$ and $\rho_{2}>0$. Then the Riemann problem for $\left(\mathscr{S}_{1} \mathscr{R}\right)$ where the initial condition is given by

$$
\mathbb{W}_{0}(x)=\left\{\begin{array}{lll}
\mathbb{W}_{L} & \text { if } & x<0 \\
\mathbb{W}_{R} & \text { if } & x>0
\end{array}\right.
$$

has a unique solution with positive densities $\rho_{k}$ for every intermediate state. The states $\mathbb{W}^{-}$and $\mathbb{W}^{+}$ respectively on the left and on the right of the standing wave are given in Appendix A.

Proof. We only sketch the proof. First of all, let us notice that from equations (17) and (18), we deduce that the densities are constant in time $\partial_{t} \rho_{k}=0$. As $\rho_{k}>0$ at time $t=0$, we get $\rho_{k}>0$ for every time $t>0$. The solution is composed of constant states separated by contact discontinuities:

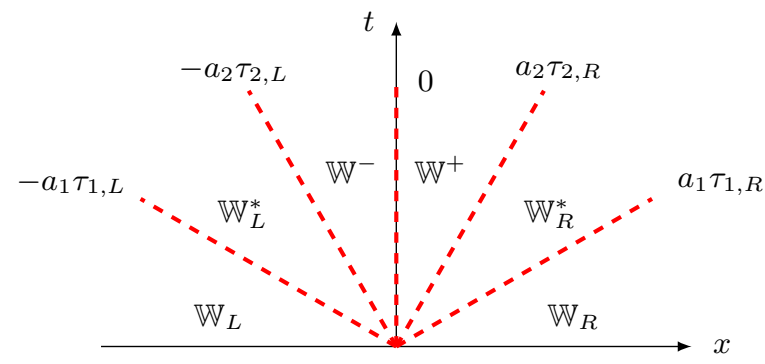

Note that the relative order of the acoustic waves $\left(a_{1} \tau_{1}<a_{2} \tau_{2}\right.$ or $\left.a_{1} \tau_{1}>a_{2} \tau_{2}\right)$ is of no importance here since it does not change the values of the intermediate states. The solution is calculated by solving a linear system of eight equations where the eight unknowns are the values of $\left(\alpha_{k} u_{k}\right)^{-}$, and $\left(\alpha_{k} \pi_{k}\right)^{-}$evaluated on the left of the standing wave as well as $\left(\alpha_{k} u_{k}\right)^{+}$, and $\left(\alpha_{k} \pi_{k}\right)^{+}$evaluated on the right of the standing wave. In order to ease the notation, we define $x_{k}:=\left(\alpha_{k} u_{k}\right)$ and $y_{k}:=\left(\alpha_{k} \pi_{k}\right)$. These quantities are linked together through the Riemann invariants of the standing wave:

$$
\begin{aligned}
& y_{1}^{-}=\phi y_{1}^{+}, \quad \text { with } \quad \phi=\alpha_{1}^{-} / \alpha_{1}^{+}, \\
& x_{2}^{-}=\psi x_{2}^{+}, \quad \text { with } \quad \psi=\alpha_{2}^{-} / \alpha_{2}^{+}, \\
& y_{1}^{-}+y_{2}^{-}=y_{1}^{+}+y_{2}^{+}, \\
& x_{1}^{-}+x_{2}^{-}=x_{1}^{+}+x_{2}^{+} .
\end{aligned}
$$

Note that $\left(\alpha_{i}^{-}, \alpha_{i}^{+}\right)=\left(\alpha_{i, L}, \alpha_{i, R}\right)$ since $\alpha_{i}$ only jumps through the standing wave. We get four additional equations by linking these unknowns with the left and right data $x_{k, L}, y_{k, L}$, and $x_{k, R}, y_{k, R}$. For example, since $u_{1}$ and $\pi_{1}$ are invariants of the $\left\{-a_{2} \tau_{2}\right\}$-wave, we have $\pi_{1}^{-}+a_{1} u_{1}^{-}=\pi_{1, L}^{*}+a_{1} u_{1, L}^{*}$. Moreover, $\pi_{1}+a_{1} u_{1}$ is a Riemann invariant of the $\left\{-a_{1} \tau_{1}\right\}$-wave which yields $\pi_{1}^{-}+a_{1} u_{1}^{-}=\pi_{1, L}^{*}+a_{1} u_{1, L}^{*}=\pi_{1, L}+a_{1} u_{1, L}$. Now, knowing that $\alpha_{1}=\alpha_{1, L}$ on the left side of the standing wave, we multiply this equation by $\alpha_{1, L}$ and we get $y_{1}^{-}+a_{1} x_{1}^{-}=y_{1, L}+a_{1} x_{1, L}$. By proceeding similarly, we get the three last equations of our system:

$$
\begin{aligned}
& y_{1}^{-}+a_{1} x_{1}^{-}=y_{1, L}+a_{1} x_{1, L}, \\
& y_{2}^{-}+a_{2} x_{2}^{-}=y_{2, L}+a_{2} x_{2, L}, \\
& y_{1}^{+}-a_{1} x_{1}^{+}=y_{1, R}-a_{1} x_{1, R}, \\
& y_{2}^{+}-a_{2} x_{2}^{+}=y_{2, R}-a_{2} x_{2, R} .
\end{aligned}
$$

Then, we can prove (see Appendix A) that up to a nonzero multiplicative constant $K$, the determinant of this linear system is equal to

$$
\operatorname{Det}=K \frac{a_{1}}{a_{2}}\left(\frac{(1-\phi)(1-\psi)}{(1+\phi)(1+\psi)} \frac{a_{1}}{a_{2}}-1\right)
$$


This determinant vanishes if and only if $\frac{(1-\phi)(1-\psi)}{(1+\phi)(1+\psi)} \frac{a_{1}}{a_{2}}-1=0$, which is impossible since

$$
\frac{(1-\phi)(1-\psi)}{(1+\phi)(1+\psi)}=-\frac{\alpha_{1}^{+}+\alpha_{1}^{-}}{\alpha_{2}^{+}+\alpha_{2}^{-}}\left(\alpha_{1}^{+}-\alpha_{1}^{-}\right)^{2} \leq 0 .
$$

Hence, the linear system is an invertible Cramer system which yields the existence and uniqueness of the solution.

\section{Numerical scheme}

Let us now describe the numerical treatment of the first step resulting from the relaxation approximation of $\left(\mathscr{S}_{1}\right)$. Starting from the given data at time $t^{n}: \mathbb{U}_{j}^{n}$, the updated data at the fictive intermediate time $t^{\sharp}$ : $\mathbb{U}_{j}^{\sharp}$ are computed as follows:

(1) Define $\mathbb{W}_{j}^{n}$ by taking the additional variables $\left(\pi_{k}\right)_{j}^{n}$ equal to $p_{k}\left(\rho_{k, j}^{n}\right)$.

(2) Apply the exact Godunov scheme to the relaxation system $\left(\mathscr{S}_{1} \mathscr{R}\right)$ with the initial data $\mathbb{W}_{j}^{n}$. At this level, the numerical parameters $a_{k}$ should be chosen, interface by interface, so as to satisfy Whitham's condition. In practice, Whitham's condition is replaced for simplicity by the following Whitham-like condition:

$$
a_{k}^{2}>\max \left(-\frac{\partial p_{k}}{\partial \tau_{k}}\left(\tau_{k, j}^{n}\right),-\frac{\partial p_{k}}{\partial \tau_{k}}\left(\tau_{k, j+1}^{n}\right)\right), \quad k=1,2 .
$$

This condition is less restrictive than the classical Whitham condition stated in (16) but it appears that in practice, no instabilities pop up in the scheme. This step yields the updated value of $\mathbb{W}_{j}^{\sharp}$.

(3) Drop the additional variable $\pi_{k}$ by taking $\mathbb{U}_{j}^{\sharp}=\left(\left(\alpha_{1}\right)_{j}^{\sharp},\left(\alpha_{1} \rho_{1}\right)_{j}^{\sharp},\left(\alpha_{1} \rho_{1} u_{1}\right)_{j}^{\sharp},\left(\alpha_{2} \rho_{2}\right)_{j}^{\sharp},\left(\alpha_{2} \rho_{2} u_{2}\right)_{j}^{\sharp}\right)$.

Points (1) to (3) provide the following finite volume scheme with non conservative numerical fluxes at the interfaces:

$$
\begin{aligned}
& \alpha_{1, j}^{\sharp}=\alpha_{1, j}^{n}, \\
& \left(\alpha_{k} \rho_{k}\right)_{j}^{\sharp}=\left(\alpha_{k} \rho_{k}\right)_{j}^{n}, \\
& \left(\alpha_{k} \rho_{k} u_{k}\right)_{j}^{\sharp}=\left(\alpha_{k} \rho_{k} u_{k}\right)_{j}^{n}-\frac{\Delta t}{\Delta x}\left(\left(\alpha_{k} \pi_{k}\right)_{j+\frac{1}{2}}^{-}-\left(\alpha_{k} \pi_{k}\right)_{j-\frac{1}{2}}^{+}\right),
\end{aligned}
$$

where $\left(\alpha_{k} \pi_{k}\right)_{j+\frac{1}{2}}^{-}$(resp. $\left.\left(\alpha_{k} \pi_{k}\right)_{j+\frac{1}{2}}^{+}\right)$are the values of $\left(\alpha_{k} \pi_{k}\right)$ on the left (resp. on the right) of the standing wave in the Riemann problem defined by $\mathbb{W}_{j}^{n}$ and $\mathbb{W}_{j+1}^{n}$ (see Appendix A for their formulae). Or course, when applying Godunov's scheme to the relaxation system, one has to restrict the time step to a classical CFL condition which reads:

$$
\frac{\Delta t}{\Delta x} \max _{j \in \mathbb{Z}} \max _{k \in\{1,2\}}\left|\left(a_{k} \tau_{k}\right)_{j}^{n}\right|<\frac{1}{2} .
$$

We have the following proposition that summarizes the properties of the relaxation numerical scheme designed for $\left(\mathscr{S}_{1}\right)$ :

Proposition 2.4. Under Whitham's condition (31) and the CFL restriction (35), equations (32)-(33)-(34) provide a numerical scheme for the first step $\left(\mathscr{S}_{1}\right)$ of the splitting method which has the following properties:

(i) It preserves the maximum principle for the phase fractions : $0<\alpha_{k}<1$, on the time step $t^{n} \rightarrow t^{\sharp}$,

(ii) It preserves positive values of the densities $\rho_{k}>0$, on the time step $t^{n} \rightarrow t^{\sharp}$,

(iii) The discretization of the partial masses $\alpha_{k} \rho_{k}$ is conservative,

(iv) The discretization of the total momentum $\alpha_{1} \rho_{1} u_{1}+\alpha_{2} \rho_{2} u_{2}$ is conservative.

Proof. The only property which is not straightforward is the conservative discretization of the total momentum. Summing equations (34) over $k$ yields:

$$
\left(\alpha_{1} \rho_{1} u_{1}+\alpha_{2} \rho_{2} u_{2}\right)_{j}^{\sharp}=\left(\alpha_{1} \rho_{1} u_{1}+\alpha_{1} \rho_{1} u_{2}\right)_{j}^{n}-\frac{\Delta t}{\Delta x}\left(\left(\alpha_{1} \pi_{1}+\alpha_{2} \pi_{2}\right)_{j+\frac{1}{2}}^{-}-\left(\alpha_{1} \pi_{1}+\alpha_{2} \pi_{2}\right)_{j-\frac{1}{2}}^{+}\right) .
$$


As $\alpha_{1} \pi_{1}+\alpha_{2} \pi_{2}$ is a Riemann invariant of the standing wave for system $\left(\mathscr{S}_{1} \mathscr{R}\right)$, we have $\left(\alpha_{1} \pi_{1}+\alpha_{2} \pi_{2}\right)_{j+\frac{1}{2}}^{-}=$ $\left(\alpha_{1} \pi_{1}+\alpha_{2} \pi_{2}\right)_{j+\frac{1}{2}}^{+}$, which preserves the conservative form.

\subsection{Treatment of the second step}

We now consider the numerical treatment of the time evolution corresponding to the second step. Starting from the output data of the first step, $\mathbb{U}_{j}^{\sharp}$, we want to compute the updated data at time $t^{n+1}: \mathbb{U}_{j}^{n+1}$.

$$
\begin{array}{ll} 
& \partial_{t} \alpha_{1}+u_{2} \partial_{x} \alpha_{1}=0, \\
\left(\mathscr{S}_{2}\right) \quad \partial_{t} \alpha_{k} \rho_{k}+\partial_{x} \alpha_{k} \rho_{k} u_{k}=0, \\
& \partial_{t} \alpha_{k} \rho_{k} u_{k}+\partial_{x} \alpha_{k} \rho_{k} u_{k}^{2}=0 .
\end{array}
$$

Equations (37) and (38) can be written in the form of two decoupled systems, each one corresponding to the material convection of mass and momentum in one of the two phases:

$$
\begin{aligned}
& \partial_{t} \alpha_{k} \rho_{k}+\partial_{x} \alpha_{k} \rho_{k} u_{k}=0, \\
& \partial_{t} \alpha_{k} \rho_{k} u_{k}+\partial_{x} \alpha_{k} \rho_{k} u_{k}^{2}=0,
\end{aligned}
$$

for $k=1$ or 2 . Each one of these two systems takes the following generic form:

$$
\begin{aligned}
& \partial_{t} \theta+\partial_{x} \theta v=0, \\
& \partial_{t} \theta \Theta+\partial_{x} \theta \Theta v=0,
\end{aligned}
$$

where $\Theta$ is a vector of $\mathbb{R}^{n}, n \geq 1$ (here $\Theta=u_{k} \in \mathbb{R}$ ) and $\theta$ is a scalar unknown that is assumed to be positive (here $\theta=\alpha_{k} \rho_{k}$ ) and for which one has to provide a scheme which preserves its positivity. Finally, $v$ is a velocity field that is assumed to depend only on the space variable: $v(x)$ (here $v(x)=u_{k}\left(t^{\sharp}, x\right)$ ). System (39) is only weakly hyperbolic, thus the numerical approximation of such a system is a priori not classical.

A positive scheme for (39)

In order to easily handle the lack of hyperbolicity, we discretize (39) with a two-step splitting operator method motivated by the following calculation:

$$
\begin{aligned}
& \partial_{t} \theta+\left\{\theta \partial_{x} v\right\}+\left\{v \partial_{x} \theta\right\}=0, \\
& \left\{\partial_{t} \theta+\partial_{x} v \theta\right\} \Theta+\theta\left\{\partial_{t} \Theta+v \partial_{x} \Theta\right\}=0 .
\end{aligned}
$$

The proposed splitting method consists in solving at first the ODE:

$$
d_{t} \theta=-\theta \frac{d}{d x} v(x)
$$

followed by

$$
\begin{aligned}
& \partial_{t} \theta+v(x) \partial_{x} \theta=0, \\
& \theta\left\{\partial_{t} \Theta+v(x) \partial_{x} \Theta\right\}=0,
\end{aligned}
$$

which can be re-written as $n+1$ decoupled transport equations:

$$
\begin{aligned}
& \partial_{t} \theta+v(x) \partial_{x} \theta=0, \\
& \partial_{t} \theta \Theta+v(x) \partial_{x} \theta \Theta=0 .
\end{aligned}
$$

The objective here is to design a time explicit discretization of (41)-(43) which is conservative for both quantities $\theta$ and $\theta \Theta$ and which preserves the positivity of $\theta$ under some natural CFL restriction. The ODE (41) is discretized with an implicit scheme as follows:

$$
\frac{\theta_{j}^{1 / 2}-\theta_{j}^{\sharp}}{\Delta t}=-\theta_{j}^{1 / 2} \frac{v_{j+\frac{1}{2}}-v_{j-\frac{1}{2}}}{\Delta x} \Longleftrightarrow \theta_{j}^{1 / 2}=\frac{\theta_{j}^{\sharp}}{1+\frac{\Delta t}{\Delta x}\left(v_{j+\frac{1}{2}}-v_{j-\frac{1}{2}}\right)} .
$$


Hence, preserving the positivity of $\theta$ in this step amounts to imposing the following CFL-like condition:

$$
1+\frac{\Delta t}{\Delta x}\left(v_{j+\frac{1}{2}}-v_{j-\frac{1}{2}}\right)>0
$$

As for the second step (43), it is discretized using the classical first order upwind scheme:

$$
\begin{aligned}
\theta_{j}^{n+1} & =\theta_{j}^{1 / 2}-\frac{\Delta t}{\Delta x}\left(\left(v_{j+\frac{1}{2}}\right)^{-}\left(\theta_{j+1}^{1 / 2}-\theta_{j}^{1 / 2}\right)+\left(v_{j-\frac{1}{2}}\right)^{+}\left(\theta_{j}^{1 / 2}-\theta_{j-1}^{1 / 2}\right)\right), \\
(\theta \Theta)_{j}^{n+1} & =\theta_{j}^{1 / 2} \Theta_{j}^{\sharp}-\frac{\Delta t}{\Delta x}\left(\left(v_{j+\frac{1}{2}}\right)^{-}\left(\theta_{j+1}^{1 / 2} \Theta_{j+1}^{\sharp}-\theta_{j}^{1 / 2} \Theta_{j}^{\sharp}\right)+\left(v_{j-\frac{1}{2}}\right)^{+}\left(\theta_{j}^{1 / 2} \Theta_{j}^{\sharp}-\theta_{j-1}^{1 / 2} \Theta_{j-1}^{\sharp}\right)\right),
\end{aligned}
$$

where for any real value $X$, we denoted $(X)^{-}=\min (0, X)$ and $(X)^{+}=\max (0, X)$. Re-writing equation (46) as

$$
\theta_{j}^{n+1}=-\frac{\Delta t}{\Delta x}\left(v_{j+\frac{1}{2}}\right)^{-} \theta_{j+1}^{1 / 2}+\left(1+\frac{\Delta t}{\Delta x}\left(\left(v_{j+\frac{1}{2}}\right)^{-}-\left(v_{j-\frac{1}{2}}\right)^{+}\right)\right) \theta_{j}^{1 / 2}+\frac{\Delta t}{\Delta x}\left(v_{j-\frac{1}{2}}\right)^{+} \theta_{j-1}^{1 / 2},
$$

we can see that this second step also preserves positive values of $\theta$ provided the following CFL condition

$$
1+\frac{\Delta t}{\Delta x}\left(\left(v_{j+\frac{1}{2}}\right)^{-}-\left(v_{j-\frac{1}{2}}\right)^{+}\right)>0 .
$$

Note that this last CFL condition may be more restrictive than (45).

We can now show that this two-step splitting operation provides a conservative discretization of (39). Injecting the result of the first step (44) in equation (46), one gets

$$
\begin{aligned}
\theta_{j}^{n+1} & =\theta_{j}^{\sharp}-\frac{\Delta t}{\Delta x} \theta_{j}^{1 / 2}\left(v_{j+\frac{1}{2}}-v_{j-\frac{1}{2}}\right)-\frac{\Delta t}{\Delta x}\left(\left(v_{j+\frac{1}{2}}\right)^{-}\left(\theta_{j+1}^{1 / 2}-\theta_{j}^{1 / 2}\right)+\left(v_{j-\frac{1}{2}}\right)^{+}\left(\theta_{j}^{1 / 2}-\theta_{j-1}^{1 / 2}\right)\right) \\
& =\theta_{j}^{\sharp}-\frac{\Delta t}{\Delta x}\left(\left(v_{j+\frac{1}{2}}\right)^{+} \theta_{j}^{1 / 2}+\left(v_{j+\frac{1}{2}}\right)^{-} \theta_{j+1}^{1 / 2}\right)+\frac{\Delta t}{\Delta x}\left(\left(v_{j-\frac{1}{2}}\right)^{+} \theta_{j-1}^{1 / 2}+\left(v_{j-\frac{1}{2}}\right)^{-} \theta_{j}^{1 / 2}\right) .
\end{aligned}
$$

This can be re-written in the following conservative form:

$$
\theta_{j}^{n+1}=\theta_{j}^{\sharp}-\frac{\Delta t}{\Delta x}\left(v_{j+\frac{1}{2}} \theta_{j+\frac{1}{2}}^{1 / 2}-v_{j-\frac{1}{2}} \theta_{j-\frac{1}{2}}^{1 / 2}\right),
$$

where for all $j$ in $\mathbb{Z}$,

$$
\theta_{j+\frac{1}{2}}^{1 / 2}= \begin{cases}\theta_{j}^{1 / 2} & \text { if } v_{j+\frac{1}{2}} \geq 0 \\ \theta_{j+1}^{1 / 2} & \text { otherwise }\end{cases}
$$

Similar calculations lead to

$$
(\theta \Theta)_{j}^{n+1}=\theta_{j}^{\sharp} \Theta_{j}^{\sharp}-\frac{\Delta t}{\Delta x}\left(v_{j+\frac{1}{2}} \theta_{j+\frac{1}{2}}^{1 / 2} \Theta_{j+\frac{1}{2}}^{\sharp}-v_{j-\frac{1}{2}} \theta_{j-\frac{1}{2}}^{1 / 2} \Theta_{j-\frac{1}{2}}^{\sharp}\right),
$$

where for all $j$ in $\mathbb{Z}$,

$$
\Theta_{j+\frac{1}{2}}^{\sharp}= \begin{cases}\Theta_{j}^{\sharp} & \text { if } v_{j+\frac{1}{2}} \geq 0, \\ \Theta_{j+1}^{\sharp} & \text { otherwise. }\end{cases}
$$

Application to equations (37) and (38)

Now, in order to apply this positive scheme to equations (37) and (38), one has to define the values of the interface velocities at the initial time: $u_{k, j+\frac{1}{2}}:=u_{k}\left(t^{\sharp}, x_{j+\frac{1}{2}}\right)$. Concerning phase number 2 , the velocity $u_{2}$ is a Riemann invariant of the standing wave in the first step $\left(\mathscr{S}_{1}\right)$. Thus, a natural choice for $u_{2, j+\frac{1}{2}}$ is $u_{2}^{-}=u_{2}^{+}$the constant value of the velocity of phase 2 across the standing wave in $\left(\mathscr{S}_{1}\right)$. One could also take 
any other consistent choice for $u_{2, j+\frac{1}{2}}$ as for instance a convex combination of $u_{2, j}^{\sharp}$ and $u_{2, j+1}^{\sharp}$ at the end of the first step:

$$
u_{2, j+\frac{1}{2}}=\beta u_{2, j}^{\sharp}+(1-\beta) u_{2, j+1}^{\sharp}, \beta \in[0,1] .
$$

As for phase 1 , whose velocity is not a Riemann invariant of the standing wave in $\left(\mathscr{S}_{1}\right)$, we decide to take:

$$
u_{1, j+\frac{1}{2}}=\eta u_{1, j}^{\sharp}+(1-\eta) u_{1, j+1}^{\sharp}, \eta \in[0,1] .
$$

In practice, we take $\beta=\eta=1 / 2$.

Finally, with this definition of $u_{2, j+\frac{1}{2}}$, the advection equation on $\alpha_{1}$ is discretized thanks to the first order upwind scheme:

$$
\alpha_{1, j}^{n+1}=\alpha_{1, j}^{\sharp}-\frac{\Delta t}{\Delta x}\left(\left(u_{2, j+\frac{1}{2}}\right)^{-}\left(\alpha_{1, j+1}^{\sharp}-\alpha_{1, j}^{\sharp}\right)+\left(u_{2, j-\frac{1}{2}}\right)^{+}\left(\alpha_{1, j}^{\sharp}-\alpha_{1, j-1}^{\sharp}\right)\right) .
$$

This discretization ensures the maximum principle on $\alpha_{1}$ if the CFL condition (49) with $v=u_{2}$ is imposed.

We have the following proposition that summarizes the properties of the relaxation numerical scheme designed for $\left(\mathscr{S}_{2}\right)$ :

Proposition 2.5. Under the CFL restriction (49), equations (44)-(46)-(47) applied to each one of the phase systems (2.3) provide a numerical scheme for the second step $\left(\mathscr{S}_{2}\right)$ of the splitting method which has the following properties:

(i) It preserves the maximum principle for the phase fractions : $0<\alpha_{k}<1$, on the time step $t^{\sharp} \rightarrow t^{n+1}$,

(ii) It preserves positive values of the densities $\rho_{k}>0$, on the time step $t^{\sharp} \rightarrow t^{n+1}$,

(iii) The discretization of the partial masses $\alpha_{k} \rho_{k}$ is conservative,

(iv) The discretization of the total momentum $\alpha_{1} \rho_{1} u_{1}+\alpha_{2} \rho_{2} u_{2}$ is conservative.

Proof. The proposition directly follows from the above discussion.

\section{NumERICAL EXPERIMENTS}

In this section, we present two test cases in which we compare the approximate solution, computed with our fractional step numerical scheme, with the exact solution of a Riemann problem. In these two cases, the phase equations of state are given by the following ideal gas pressure laws:

$$
\begin{aligned}
& p_{1}\left(\rho_{1}\right)=\kappa_{1} \rho_{1}^{\gamma_{1}}, \quad \text { with } \kappa_{1}=1 \text { and } \gamma_{1}=3, \\
& p_{2}\left(\rho_{2}\right)=\kappa_{2} \rho_{2}^{\gamma_{2}}, \quad \text { with } \kappa_{2}=1 \text { and } \gamma_{2}=1.5 .
\end{aligned}
$$

The solutions are computed on the domain $[-0.5,0.5]$ of the $x$-space. For both tests 1 and 2 , a mesh refinement process is implemented in order to numerically check the convergence of the method. For this purpose, we compute the discrete $L^{1}$-error between the approximate solution and the exact one at the final time $T$, normalized by the discrete $L^{1}$-norm of the exact solution:

$$
\operatorname{error}(\Delta x)=\frac{\sum_{\text {cells } j}\left|\mathcal{U}_{j}^{n}-\mathcal{U}_{e x}\left(x_{j}, T\right)\right| \Delta x}{\sum_{\text {cells } j}\left|\mathcal{U}_{e x}\left(x_{j}, T\right)\right| \Delta x}
$$

where $\mathcal{U}$ denotes the state vector in non conservative variables:

$$
\mathcal{U}=\left(\alpha_{1}, \rho_{1}, u_{1}, \rho_{2}, u_{2}\right)
$$

The calculations have been implemented on several meshes. The coarser mesh is composed of 100 cells and the more refined one contains 200000 cells. The error error $(\Delta x)$ is then plotted against $\Delta x$ in a $\log -\log$ scale.

Notations: 
- $\mathcal{R}_{\sigma_{i}}\left(\mathcal{U}^{-}, \mathcal{U}^{+}\right)$stands for a $\sigma_{i}$-rarefaction wave, $i=2,3,4,5$, connecting the left-hand state $\mathcal{U}^{-}$to the right-hand state $\mathcal{U}^{+}$.

- $\mathcal{S}_{\sigma_{i}}\left(\mathcal{U}^{-}, \mathcal{U}^{+}\right)$stands for a $\sigma_{i}$-shock, $i=2,3,4,5$, connecting the left-hand state $\mathcal{U}^{-}$to the right-hand state $\mathcal{U}^{+}$.

- $\mathcal{C}_{\sigma_{1}}\left(\mathcal{U}^{-}, \mathcal{U}^{+}\right)$stands for a $\sigma_{1}$-contact discontinuity connecting the left-hand state $\mathcal{U}^{-}$to the right-hand state $\mathcal{U}^{+}$.

Finally, a $\sigma_{i}$-wave connecting a state $\mathcal{U}_{1}$ to a state $\mathcal{U}_{2}$ followed by a $\sigma_{j}$-wave connecting $\mathcal{U}_{2}$ to $\mathcal{U}_{3}$ will be denoted $\mathcal{W}_{\sigma_{i}}\left(\mathcal{U}_{1}, \mathcal{U}_{2}\right) \longrightarrow \mathcal{W}_{\sigma_{j}}\left(\mathcal{U}_{2}, \mathcal{U}_{3}\right), \mathcal{W}=\mathcal{R}, \mathcal{S}, \mathcal{C}$

\subsection{Test case 1: a contact discontinuity}

The first test case is a Riemann problem with only a $\sigma_{1}=u_{2}$ contact discontinuity. In the exact solution, all the physical quantities are transported with the constant velocity $u_{2}=0.1$, except $u_{2}$ which is constant. The initial data in non conservative variables is defined as

$$
\begin{array}{ll}
\mathcal{U}_{L}=(0.3,1 ., 0.2,0.8,0.1) & \text { for } x<0 \\
\mathcal{U}_{R}=(0.6,1.0012502584,0.1499375651,0.6302289018,0.1) & \text { for } x>0 .
\end{array}
$$

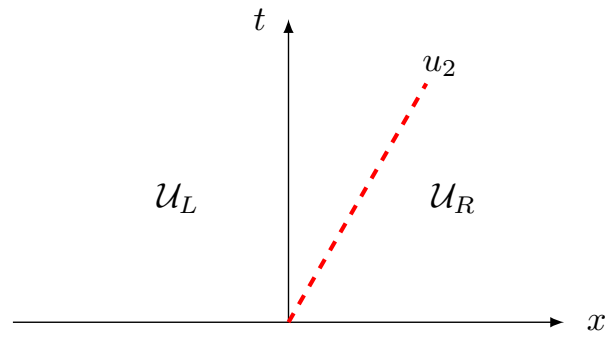

Wave structure of the exact Riemann solution

Figure 1 shows that the moving contact is not exactly captured by our scheme. However, when the exact solution of a Riemann problem contains a contact discontinuity, the expected order of convergence in $L^{1}$-norm is $\Delta x^{1 / 2}$ for a first order scheme. In Figure 1, we can see that our splitting method provides convergence towards the exact solution with the expected order of $\Delta x^{1 / 2}$. Note that, to our knowledge, there exists no solver that is able to capture exactly a moving contact discontinuity on coarse meshes, and our scheme compares rather well with other schemes (see [13]). Nevertheless, the method proposed in [16] exactly captures stationary contacts, i.e. contacts with $u_{2}=0$.

The strange behavior of the scheme on the density variable of phase 1 is due to the present choice of initial conditions on $\rho_{1}$ in which the left and right values are very close. This makes the jump more difficult to be captured on this variable, and it is all the more difficult for coarse meshes.

Finally, we would like the reader to be aware that the number of visible points in the figure for the 50000-cell mesh (especially in the contact wave) is not the real one since some points have been dropped for the clarity of the graph.

\subsection{Test case 2: a complete case with all the waves}

The second test case is a complete case with the contact discontinuity and all the acoustic waves. The initial data in non conservative variables is set to

$$
\begin{array}{ll}
\mathcal{U}_{L}=(0.1,0.85,0.4609513139,0.96,0.0839315299) & \text { for } x<0 \\
\mathcal{U}_{R}=(0.6,1.2520240113,0.7170741165,0.2505659851,-0.3764790609) & \text { for } x>0
\end{array}
$$


Error in L1-norm
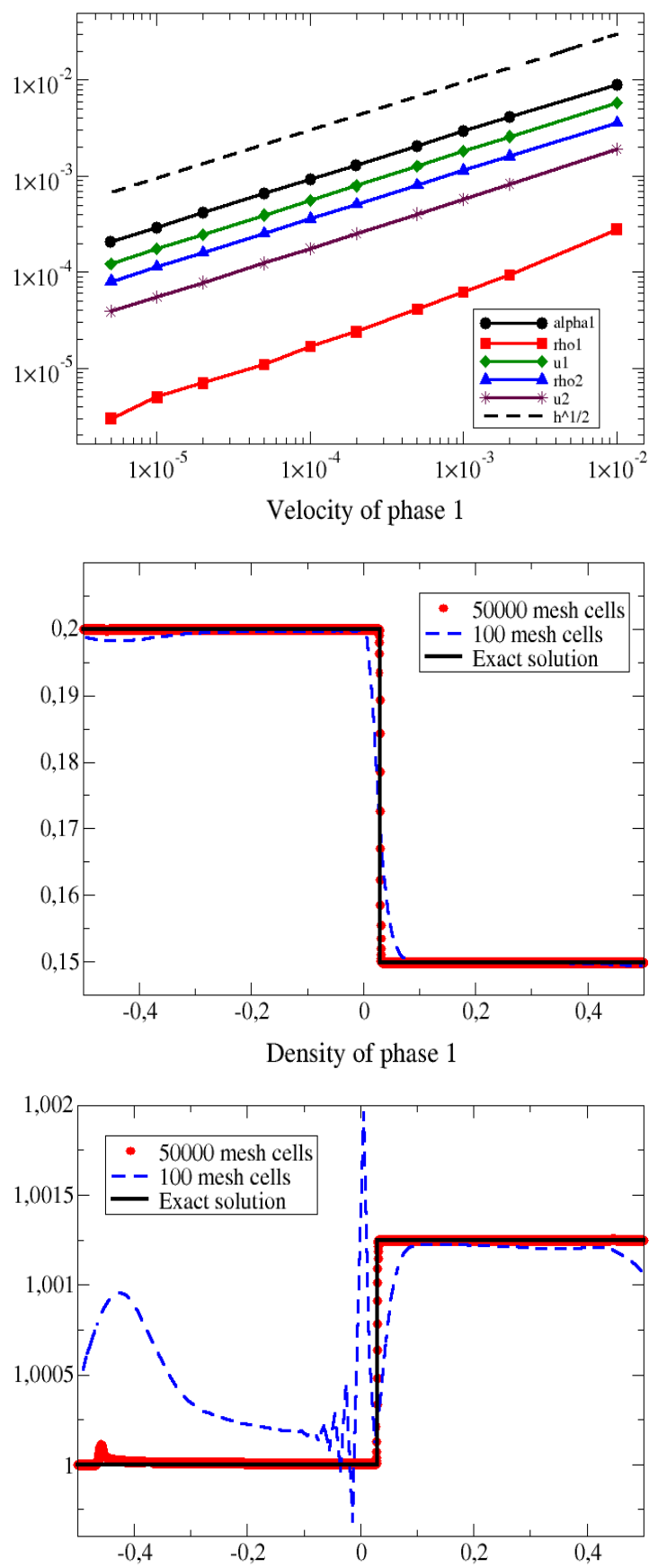

Fraction of phase 1
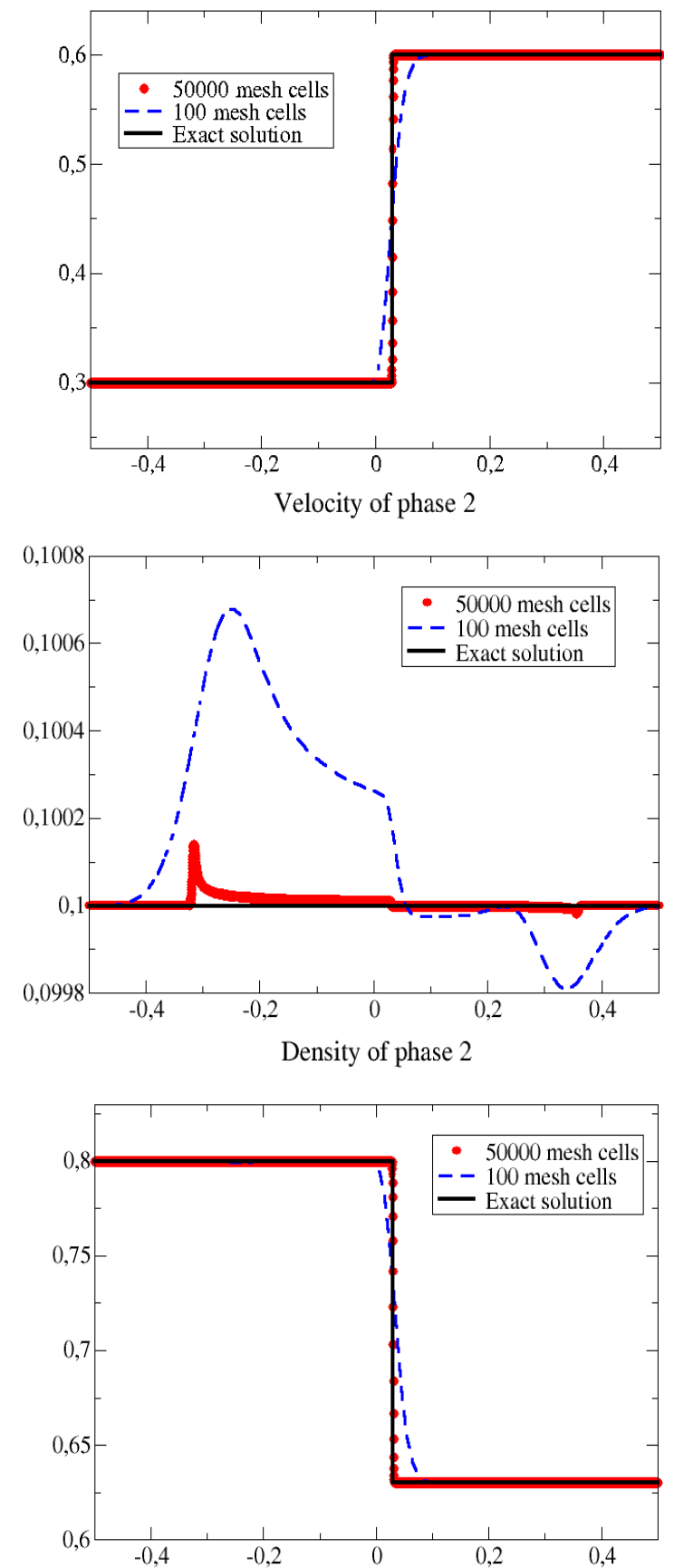

Figure 1. Test 1: space variations of the physical variables at the final time $T=0.3$, and $L^{1}$-norm of the error for several mesh sizes.

Observe that this is a hard case to run since the difference between the left and right values of the phase fraction $\alpha_{1}$ is quite large. The intermediate states (also in non conservative variables) are given by:

$$
\begin{aligned}
& \mathcal{U}_{1}=(0.1,1 ., 0.2,0.96,0.0839315299), \\
& \mathcal{U}_{2}=(0.1,1 ., 0.2,0.8,0.3), \\
& \mathcal{U}_{3}=(0.6,1.0016192090,0.2833602765,0.5011319701,0.3), \\
& \mathcal{U}_{4}=(0.6,1.0016192090,0.2833602 \mathfrak{F} 65,0.2505659851,-0.3764790609) .
\end{aligned}
$$


The Riemann solution is a $\left\{u_{1}-c_{1}\right\}$-shock wave from $\mathcal{U}_{L}$ to $\mathcal{U}_{1}$, followed by a $\left\{u_{2}-c_{2}\right\}$-rarefaction wave from $\mathcal{U}_{1}$ to $\mathcal{U}_{2}$, followed by a $u_{2}$-contact discontinuity from $\mathcal{U}_{2}$ to $\mathcal{U}_{3}$, followed by a $\left\{u_{2}+c_{2}\right\}$-shock from $\mathcal{U}_{3}$ to $\mathcal{U}_{4}$ and finally followed by a $\left\{u_{1}+c_{1}\right\}$-rarefaction wave from $\mathcal{U}_{4}$ to $\mathcal{U}_{R}$ :

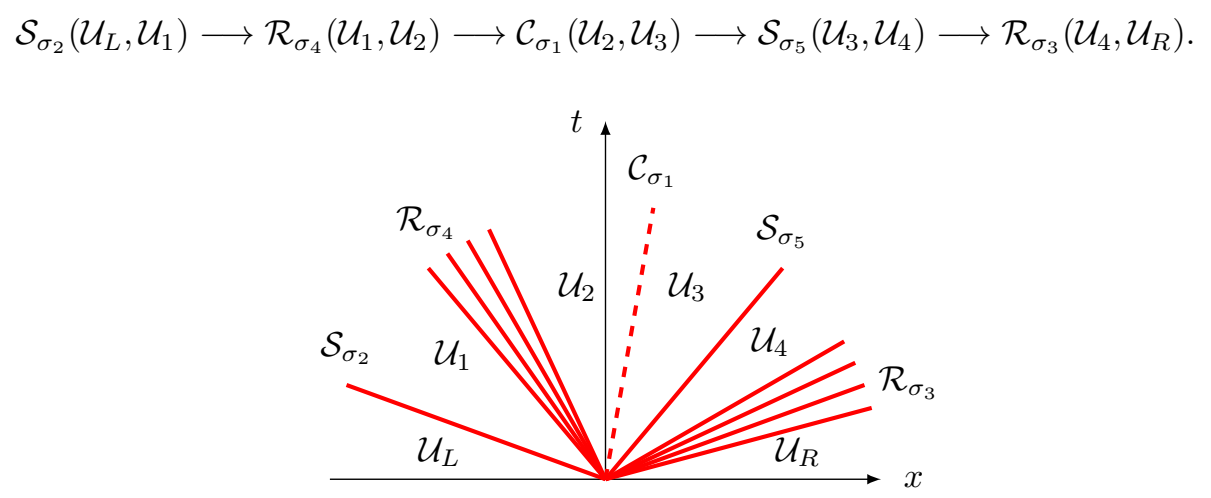

Wave structure of the exact Riemann solution

In Figure 2, we can see that the intermediate states are correctly captured by the method even for a quite coarse mesh of 100 cells. Figure 2 also shows that the approximate solution computed thanks to the splitting method converges towards the exact solution. Only the error on the phase fraction $\alpha_{1}$ converges towards zero with the expected order of $\Delta x^{1 / 2}$, while the other variables seem to converge with a higher rate. However, the expected order of $\Delta x^{1 / 2}$ is only an asymptotic order of convergence, and in this particular case, one would have to implement the calculation on much more refined meshes in order to recover this expected order $\Delta x^{1 / 2}$.

Here again, we warn the reader that the number of visible points in the figure for the 50000-mesh (especially in the contact wave and in the shocks) is not the real one since some points have been dropped for the clarity of the graph.

\section{Conclusion}

The explicit scheme presented here provides convergent approximations of discontinuous solutions of the barotropic Baer-Nunziato model, while preserving the maximum principle on the values of the statistical fractions $\alpha_{k}$ and positive values of the densities $\rho_{k}$. A sequel of this work consists in using the same fractional step strategy in order to derive an implicit version of the first step, and thus to get rid of a rather constraining CFL condition due to the propagation of fast acoustic waves.

\section{A. Proof of Proposition 2.3 And formulae of the intermediate states $\mathbb{W}^{-}$ AND $\mathbb{W}^{+}$}

We prove here that the determinant of the system composed of equations (22) to (29) has the following expression

$$
\operatorname{Det}=K \frac{a_{1}}{a_{2}}\left(\frac{(1-\phi)(1-\psi)}{(1+\phi)(1+\psi)} \frac{a_{1}}{a_{2}}-1\right)
$$

for some constant $K \neq 0$. We first ease the notations by denoting the data in the right hand side part of equations (26) to (29) as follows:

$$
\begin{aligned}
& A:=y_{1, L}+a_{1} x_{1, L}, \\
& B:=y_{2, L}+a_{2} x_{2, L}, \\
& C:=y_{1, R}-a_{1} x_{1, R}, \\
& D:=y_{2, R}-a_{2} x_{2, R} .
\end{aligned}
$$


Error in L1-norm
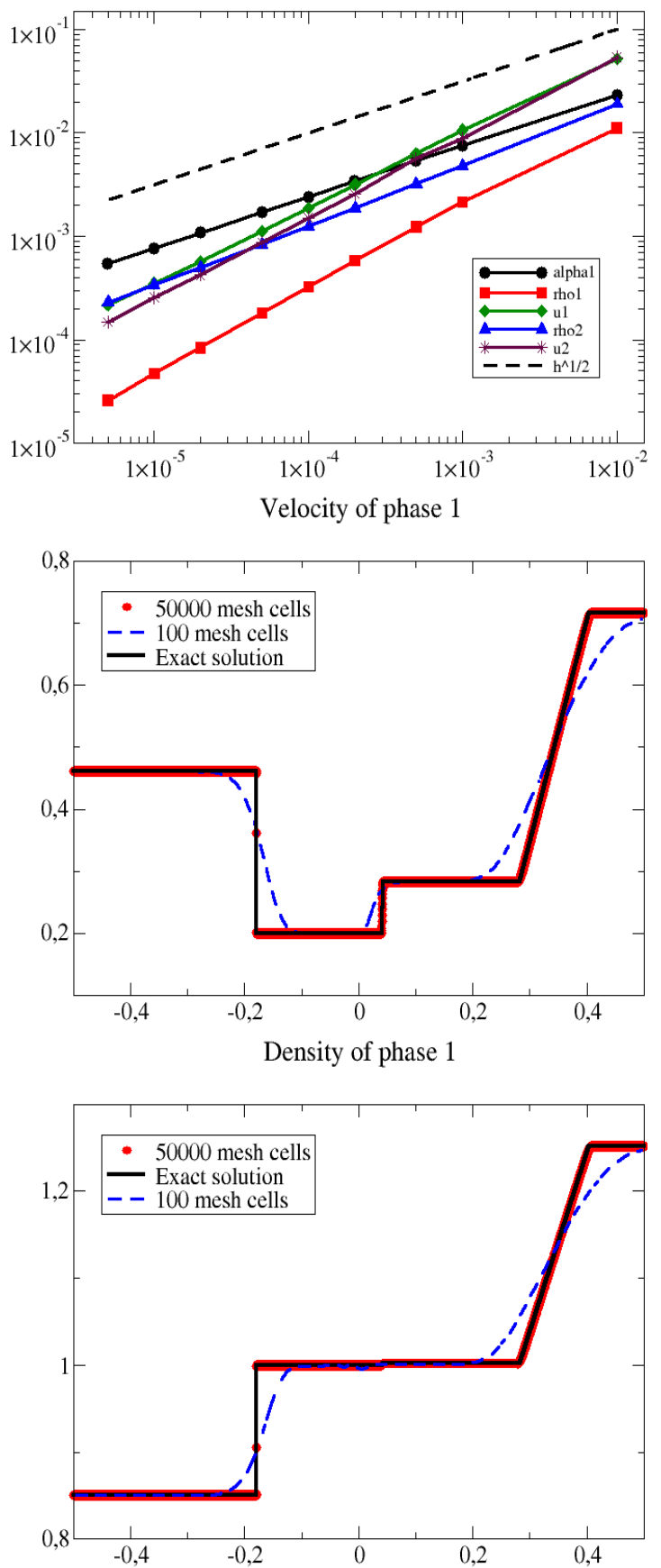

Fraction of phase 1
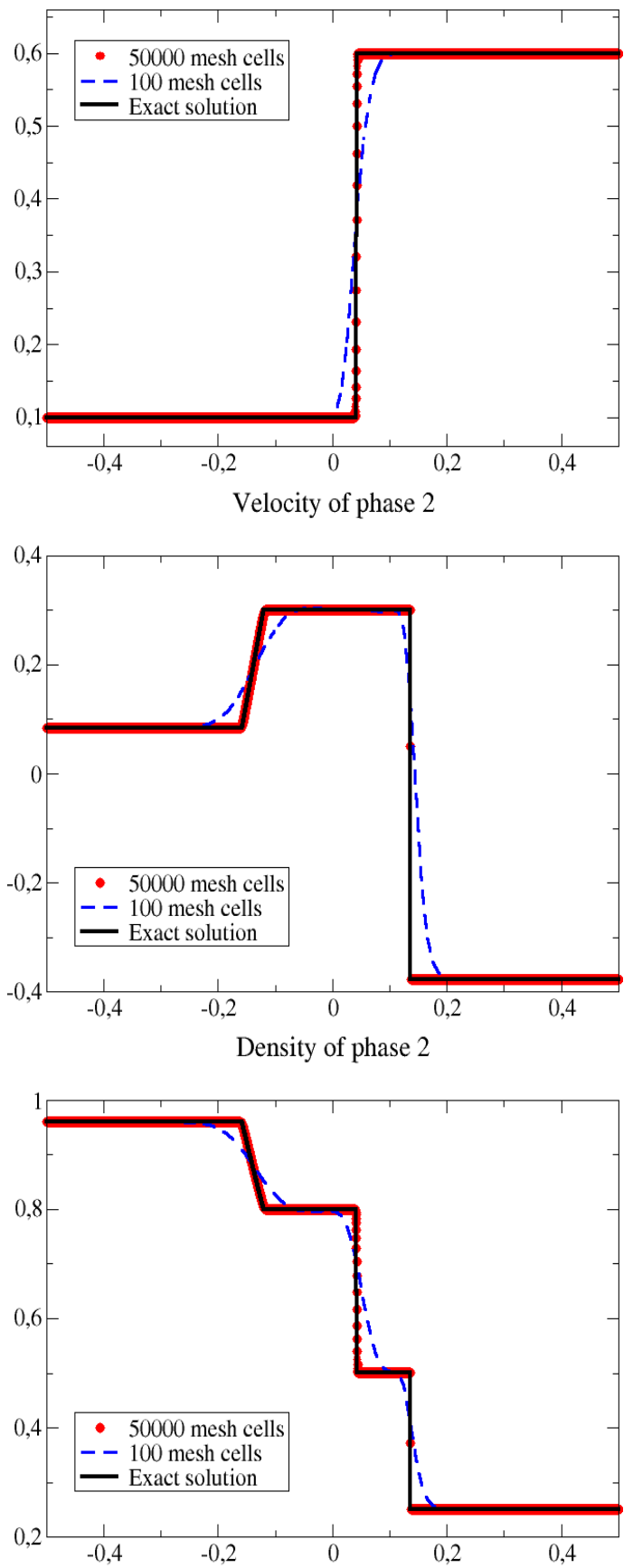

Figure 2. Test 2: space variations of the physical variables at the final time $T=0.14$, and $L^{1}$-norm of the error for several mesh sizes.

We also denote $u=x_{1}^{-}$and $v=x_{1}^{+}$. We express all the other unknowns in terms of $u$ and $v$ in order to bring this eight equation linear system to a system of two linear equations on $u$ and $v$. We note that provided that $a_{2} \neq 0$, equations (24), (25), (62), (63), (64) and (65) form an autonomous system that can be solved with respect to $u$ and $v$ and whose solution is 


$$
\begin{aligned}
& y_{1}^{-}=A-a_{1} u, \\
& y_{1}^{+}=C+a_{1} v, \\
& y_{2}^{-}=\frac{a_{1}+a_{2}}{2} u+\frac{a_{1}-a_{2}}{2} v+\frac{B+C+D-A}{2}, \\
& y_{2}^{+}=\frac{a_{2}-a_{1}}{2} u-\frac{a_{1}+a_{2}}{2} v+\frac{A+B+D-C}{2}, \\
& x_{2}^{-}=-\frac{1}{2}\left(1+\frac{a_{1}}{a_{2}}\right) u+\frac{1}{2}\left(1-\frac{a_{1}}{a_{2}}\right) v+\frac{A+B-C-D}{2 a_{2}}, \\
& x_{2}^{+}=\frac{1}{2}\left(1-\frac{a_{1}}{a_{2}}\right) u-\frac{1}{2}\left(1+\frac{a_{1}}{a_{2}}\right) v+\frac{A+B-C-D}{2 a_{2}} .
\end{aligned}
$$

Denoting $X=a_{1} / a_{2}$ and injecting these expressions in equation (22) and (23), we get

$$
\frac{A}{a_{2}}-X u=\phi\left(\frac{C}{a_{2}}+X v\right) \Longleftrightarrow X u+\phi X v=\frac{A-\phi C}{a_{2}},
$$

and

$$
\begin{aligned}
& -(1+X) u+(1-X) v+\frac{A+B-C-D}{a_{2}}=\psi\left((1-X) u-(1+X) v+\frac{A+B-C-D}{a_{2}}\right) \\
& \Longleftrightarrow(\psi(1-X)+1+X) u+(X-1-\psi(1+X)) v=(1-\psi) \frac{A+B-C-D}{a_{2}} .
\end{aligned}
$$

Equations (72) and (73) form a $2 \times 2$ linear system whose determinant is

$$
\begin{aligned}
\text { Det } & =X(X-1-\psi(1+X))-\phi X(\psi(1-X)+1+X) \\
& =(1-\phi)(1-\psi) X^{2}-(1+\phi)(1+\psi) X \\
& =(1+\phi)(1+\psi) X\left(\frac{(1-\phi)(1-\psi)}{(1+\phi)(1+\psi)} X-1\right),
\end{aligned}
$$

which proves the expression of the determinant given in equation (30). Hence, using Cramer formulae, we deduce the expressions of $u$ and $v$ :

$$
\begin{aligned}
& u=\frac{1}{\operatorname{Det}}\left(\frac{A-\phi C}{a_{2}}(X-1-\psi(1+X))-(1-\psi) \frac{A+B-C-D}{a_{2}} \phi X\right), \\
& v=\frac{1}{\operatorname{Det}}\left(X(1-\psi) \frac{A+B-C-D}{a_{2}}-\frac{A-\phi C}{a_{2}}(\psi(1-X)+1+X)\right),
\end{aligned}
$$

which yields $x_{1}^{-}=u, x_{1}^{+}=v$. The formulae of $y_{1}^{-}, y_{1}^{+}, y_{2}^{-}, y_{2}^{+}, x_{2}^{-}$and $x_{2}^{+}$are given in equations (66) to (71).

\section{REFERENCES}

[1] A. Ambroso, C. Chalons, F. Coquel, and T. Galié. Relaxation and numerical approximation of a two-fluid two-pressure diphasic model. M2AN Math. Model. Numer. Anal., 43(6):1063-1097, 2009.

[2] M.R. Baer and J.W. Nunziato. A two-phase mixture theory for the deflagration-to-detonation transition (DDT) in reactive granular materials. International Journal of Multiphase Flow, 12(6):861 - 889, 1986.

[3] R. Baraille. Dévelopement de schémas numériques adaptés à l'hydrodynamique. PhD thesis, Université Bordeaux I, 1991.

[4] D. Bresch, B. Desjardins, J. Ghidaglia, and E. Grenier. Global weak solutions to a generic two-fluid model. Archive for Rational Mechanics and Analysis, 196:599-629, 2010.

[5] C. Chalons, F. Coquel, S. Kokh, and N. Spillane. Large time-step numerical scheme for the seven-equation model of compressible two-phase flows. Springer Proceedings in Mathematics, FVCA 6, 2011, 4:225-233, 2011. 
[6] F. Coquel, T. Gallouët, J-M. Hérard, and N. Seguin. Closure laws for a two-fluid two-pressure model. C. R. Math. Acad. Sci. Paris, 334(10):927-932, 2002.

[7] F. Coquel, E. Godlewski, and N. Seguin. Relaxation of fluid systems. Math. Models Methods Appl. Sci., $22(8), 2012$.

[8] P. Embid and M. Baer. Mathematical analysis of a two-phase continuum mixture theory. Contin. Mech. Thermodyn., 4(4):279-312, 1992.

[9] S. Gavrilyuk and R. Saurel. Mathematical and numerical modeling of two-phase compressible flows with micro-inertia. Journal of Computational Physics, 175(1):326 - 360, 2002.

[10] J-M. Hérard and O. Hurisse. A fractional step method to compute a class of compressible gas-luiquid flows. Computers 86 Fluids. An International Journal, 55:57-69, 2012

[11] A. K. Kapila, S. F. Son, J. B. Bdzil, R. Menikoff, and D. S. Stewart. Two-phase modeling of DDT: Structure of the velocity-relaxation zone. Physics of Fluids, 9(12):3885-3897, 1997.

[12] S. Karni and G. Hernàndez-Dueñas. A hybrid algorithm for the Baer-Nunziato model using the Riemann invariants. Journal of Scientific Computing, 45:382-403, 2010.

[13] Y. Liu. PhD thesis, Université Aix-Marseille, to appear in 2013.

[14] R. Saurel and R. Abgrall. A multiphase godunov method for compressible multifluid and multiphase flows. Journal of Computational Physics, 150(2):425 - 467, 1999.

[15] D.W. Schwendeman, C.W. Wahle, and A.K. Kapila. The Riemann problem and a high-resolution Godunov method for a model of compressible two-phase flow. Journal of Computational Physics, 212(2):490 - 526, 2006.

[16] M. D. Thanh, D. Kröner, and N. T. Nam. Numerical approximation for a Baer-Nunziato model of two-phase flows. Applied Numerical Mathematics, 61(5):702 - 721, 2011.

[17] S.A. Tokareva and E.F. Toro. HLLC-type Riemann solver for the Baer-Nunziato equations of compressible two-phase flow. Journal of Computational Physics, 229(10):3573 - 3604, 2010. 